\title{
Les glucides et les lipides du clitellum et de l'albumen du cocon chez Eisenia fetida Sav (annélide oligochète)
}

\author{
L Rouabah-Sadaoui *, R Marcel ** \\ Laboratoire d'écophysiologie d'invertébrés du sol, université des sciences et technologies de Lille, \\ 59655 Villeneuve-d'Ascq cedex, France
}

(Reçu le 25 janvier 1995 ; accepté le 7 juillet 1995)

\begin{abstract}
Résumé - L'analyse des constituants glucidiques et lipidiques de l'épithélium clitellaire et de l'albumen des cocons est abordée à l'aide de techniques biochimiques chez Eisenia fetida. La teneur en glucides augmente considérablement (environ $\times 5$ ) au cours de la différenciation clitellaire. Les oses et les osides représentent $50 \%$ du poids sec des cocons fraîchement pondus. On note un enrichissement en certains hexoses lors de la puberté. Le glucose prédomine chez les vers immatures, le mannose dans les clitellums mûrs. L'approche de l'éclosion s'accompagne d'une baisse générale de la teneur de tous les glucides. La teneur en lipides totaux du clitellum augmente au cours de la maturation : celle des lipides neutres, les plus abondants, varie peu ; en revanche, celle des phospholipides, beaucoup plus rares, est en notable augmentation. Le taux des acides gras du clitellum présente un maximum à la puberté ; le cocon accumule une grande quantité d'acides gras. Les acides palmitique, stéarique, oléique et vaccénique sont prépondérants.
\end{abstract}

\section{glucide / lipide / Eisenia / ver de terre}

Summary - Glucids and lipids of clitellum and cocoon's albumen in Eisenia fetida Sav (Annelida Oligochaeta). Biochemical methods were used to analyze the glucidic and lipidic components of the clitellar epithelium and the cocoon's albumen in Eisenia fetida. In the clitellum, the sugar concentration increased dramatically (about 5-fold) during differentiation. The assays showed increases in several monosaccharides during puberty. In addition, glucose was prominent in immature clitella, whereas mannose was the main sugar in the mature ones. About $50 \%$ of the dry weight of recently deposited cocoons was carbohydrates. The sugar concentration decreased in the albumen as hatching approached. The amount of total lipids in the clitellum increased during maturation. The levels of neutral lipids, however varied very little. Phospholipids began at low levels at the beginning of differentiation, and

* Nouvelle adresse : Institut des sciences biologiques, université de Constantine, Constantine, Algérie

** Correspondance et tirés à part 
increased dramatically thereafter. The amount of fatty acids in the clitellar epithelium reached a maximum during the submature stage. Large amounts of fatty acids were stored in the cocoon. Palmitic, stearic, oleic and vaccenic acids were preponderant in both the clitellum and cocoon.

glucid / lipid / Eisenia / earthworm

\section{INTRODUCTION}

Le cocon des lombriciens, sécrété par le clitellum, renferme des œufs oligolécithes (Dawydoff, 1959) dont le cytoplasme ne contient qu'une petite quantité de produits lipidiques utilisés pendant la segmentation de l'embryon (Lechenault, 1968). Les éléments nutritifs de l'albumen du cocon sont consommés à partir du stade gastrula (Devriès, 1973). Trois catégories cellulaires sont impliquées dans la formation du cocon : les "cellules à mucus», les «cellules à gros granules», et les "cellules à fins granules» qui sont responsables de la sécrétion de l'albumen (Grove et Cowley, 1928).

La nature des différents éléments cellulaires du clitellum a été étudiée par des méthodes histochimiques (Defretin et Demailly, 1953). L'analyse chimique a été abordée par des méthodes biochimiques appliquées à l'animal entier (Durchon et Lafon, 1951 ; De Jorge et al, 1965 ; De Jorge et Sawaya, 1967) ou au clitellum uniquement (Rouabah-Sadaoui, 1984).

Dans une note précédente (RouabahSadaoui et Marcel, 1995), nous avons mis en évidence, dans les clitellums et dans l'albumen des cocons, une glycolipoprotéine, assimilable à une vitellogénine, ainsi que 2 glycoprotéines. À côté des protéines, les glucides et les lipides jouent donc un rôle important dans la constitution des réserves destinées au développement de l'embryon. Nous nous sommes proposés d'étudier la composition glucidique et lipidique des clitellums et de l'albumen des cocons, pour faire suite à l'analyse des protéines.

\section{MATÉRIEL ET MÉTHODES}

Les Eisenia, élevés au laboratoire, ont été récoltés à 3 stades de maturation sexuelle :

- immatures, ne présentant aucune différenciation clitellaire apparente :

- submatures, dont la région où se développera le clitellum montre une coloration plus claire caractéristique ;

- mûrs, présentant un clitellum turgescent.

Les clitellums, réséqués sous anesthésie au chloréthone à $0,2 \%$, ont été broyés dans l'eau distillée avec un homogénéiseur Dounce. Après centrifugation à $2900 \mathrm{~g}$, pendant $10 \mathrm{~min}$, le surnageant a été lyophilisé.

Les clitellums et les cocons lyophilisés ont été pesés au préalable, pour en déterminer les poids secs, sur une balance fermée, en atmosphère anhydre, en présence de potasse.

Les cocons pondus par des vers sexuellement actifs ont été récoltés tous les jours, puis mis en incubation à $24^{\circ} \mathrm{C}$. Ces cocons ont été ouverts à différents moments : $1,5,10,15$ et $20 \mathrm{j}$ après la ponte et leur contenu (albumen et embryons) séparé de l'enveloppe, puis lyophilisé.

Les oses neutres totaux sont dosés sur les lyophilisats (surnageant de l'homogénat des clitellums et contenu des cocons) par la méthode à l'orcinol sulfurique de Tillmans et Philippi modifiée par Rimigton, d'après Montreuil et Spik (1963). Les oses et hexosamines sont identifiés et dosés par chromatographie en phase gazeuse des trifluoroacétylés selon la technique de Zanetta et al (1972). La fraction à étudier, additionnée de mésoinositol (témoin interne), est lyophilisée puis méthanolysée (méthanol $+\mathrm{HCl}$ à $0,5 \mathrm{~N}: 1 \mathrm{~mL}$ par $\mathrm{mg}$ de produit) à $80^{\circ} \mathrm{C}$ pendant $24 \mathrm{~h}$. Les protéines sont éliminées par centrifugation tandis que les lipides sont dissous dans l'heptane. La trifluoroacétylation (dichlorométhane $100 \mu \mathrm{L}$ anhydride trifluoroacétique $100 \mu \mathrm{L}$ ) est réalisée à $150^{\circ} \mathrm{C}$ au bain de sable. La chromatographie est exécutée sur colonne de verre $(0,3 \times 300 \mathrm{~cm})$ 
OV 210 ; gaz vecteur : azote à $15 \mathrm{~mL} / \mathrm{min}$; température programmée de $100^{\circ} \mathrm{C}$ à $200^{\circ} \mathrm{C}$ à raison de $2^{\circ} \mathrm{C} / \mathrm{min}$; température de l'injecteur et du détecteur $220^{\circ} \mathrm{C}$.

Les lipides totaux sont extraits des différents échantillons par le mélange chloroforme : méthanol $(2: 1 \mathrm{~V} / \mathrm{N})$, suivant la méthode de Folch et al (1957). Les substances non lipidiques sont éliminées par addition d'une solution aqueuse à $9 \%$ de $\mathrm{Na} \mathrm{Cl}$, à raison de 1 volume pour 5 volumes d'extrait lipidique. Après agitation, le déphasage s'opère dans une ampoule à décanter, pendant une nuit, à $+4^{\circ} \mathrm{C}$. Les lipides totaux, purifiés, restent en solution dans la phase chloroformique supérieure. Dans cette dernière, les phospholipides sont séparés des lipides neutres par précipitation acétonique et centrifugation. Le surnageant acétonique est séché sous courant d'azote, puis lyophilisé. Les lipides neutres sont alors saponifiés par la soude $4 \mathrm{~N}$, à $100^{\circ} \mathrm{C}$, pendant $4 \mathrm{~h}$. Les acides gras sont dissous dans du chloroforme et lyophilisés avant d'être estérifiés $\left(80^{\circ} \mathrm{C}\right.$, $4 \mathrm{~h}$ ) par du méthanol chlorhydrique. Après séchage sous courant d'azote, le résidu est repris par de l'heptane et les méthylesters d'acides gras sont analysés par chromatographie en phase gazeuse (Girdel série 300 ) sur colonne capillaire de silicone OV 101 ( $85 \mathrm{~m} \mathrm{x} \mathrm{0,35} \mathrm{mm} \mathrm{ID)} \mathrm{;} \mathrm{pres-}$ sion du gaz vecteur (hélium) : 0,4 bar ; température programmée de $100^{\circ} \mathrm{C}$ à $200^{\circ} \mathrm{C}$ à raison de $2^{\circ} \mathrm{C}$ par min ; température de l'injecteur et du détecteur : $240^{\circ} \mathrm{C}$. L'identification des acides gras est confirmée par couplage chromatographie en phase gazeuse - spectrométrie de masse (GLC MS) à l'aide d'un appareil Riber-Mag $R$ 10-10 ; potentiel d'ionisation $70 \mathrm{eV}$; gamme de masses : $70-250$ en $400 \mathrm{~ms}$.

Le traitement statistique des résultats est effectué à l'aide du test t de Student.

\section{RÉSULTATS}

\section{Les glucides}

\section{Dosages des oses totaux par colorimétrie à l'orcinol}

Les résultats des dosages, exprimés en pourcentage du poids sec, sont consignés dans le tableau I. La teneur en glucides, faible dans le clitellum immature $(4,5 \%)$ augmente considérablement au cours de la différenciation clitellaire $(21 \%$ dans le clitellum turgescent). Une décroissance des taux de glucides totaux dans les cocons (albumen + embryons) est observée au cours de l'embryogenèse (tableau II) : de $25-30 \%$, pendant les 10 premiers jours, à $15 \%$, dans les cocons proches de l'éclosion. Toutes ces différences sont statistiquement significatives $(P \leq 0,01)$.

\section{Identification et dosage des oses neutres par chromatographie en phase gazeuse}

Les résultats précédents, acquis sur les glucides totaux, nous ont incités à rechercher leur composition dans le clitellum et dans les cocons. Nous avons pu ainsi identifier (fig 1) et doser les oses neutres suivants : galactose (Gal), glucose (Glc), mannose (Man), fucose (Fuc), xylose (Xyl). Les hexosamines, glucosamine (GlcNac) et galactosamine (GalNac), ont été identifiés et dosés. Les acides glucuronique et sialique n'ont pas été décelés.

Les résultats des dosages sont exprimés, dans le tableau III, sous 2 formes : en pourcentage par rapport au poids sec et en pourcentage par rapport aux glucides totaux. Ce tableau montre que les teneurs de tous les

Tableau I. Teneurs en oses neutres totaux, exprimées en \% par rapport au poids sec (méthode à l'orcinol).

\section{Clitellums}

Immatures Submatures Mûrs

$$
4,5 \pm 1,8 \quad 11,8 \pm 1,7 \quad 21,0 \pm 1,8
$$

Poids sec moyen d'un clitellum mûr : $1,02 \pm 0,05 \mathrm{mg}$, submature : $0,89 \pm 0,03 \mathrm{mg}$, immature : $0,78 \pm$ $0,03 \mathrm{mg}$. 
Tableau II. Teneurs des cocons en oses neutres totaux, exprimées en \% par rapport au poids sec (méthode à l'orcinol), au cours du développement embryonnaire.

Albumen des cocons de
$1 j$
$5 j$
$10 j$
$15 j$
$20 j$
$30,0 \pm 1,6$
$24,7 \pm 1,6$
$31,8 \pm 1,3$
$23,5 \pm 1,4$
$15,4 \pm 1,4$

Poids sec moyen d'un cocon frais pondu : $0,91 \pm 0,06 \mathrm{mg}$.

glucides augmentent lors de la maturation du clitellum, en particulier le galactose, le mannose et les hexosamines. En revanche, le fucose et le xylose demeurent à des concentrations relativement faibles. L'expression du pourcentage de ces glucides par rapport aux glucides totaux permet de mettre en évidence la prédominance du glucose chez les vers immatures et celle du mannose dans les clitellums mûrs. Les résultats du tableau III exprimant les teneurs des glucides totaux d'un lot de cocons de moins de $10 \mathrm{j}$ (dernière colonne) confirment donc les résultats des dosages à l'orcinol (tableau I). Les cocons contiennent au moins 2 fois plus de glucides que le clitellum mûr. Clitellum et albumen possèdent les mêmes oses. Le taux du mannose demeure

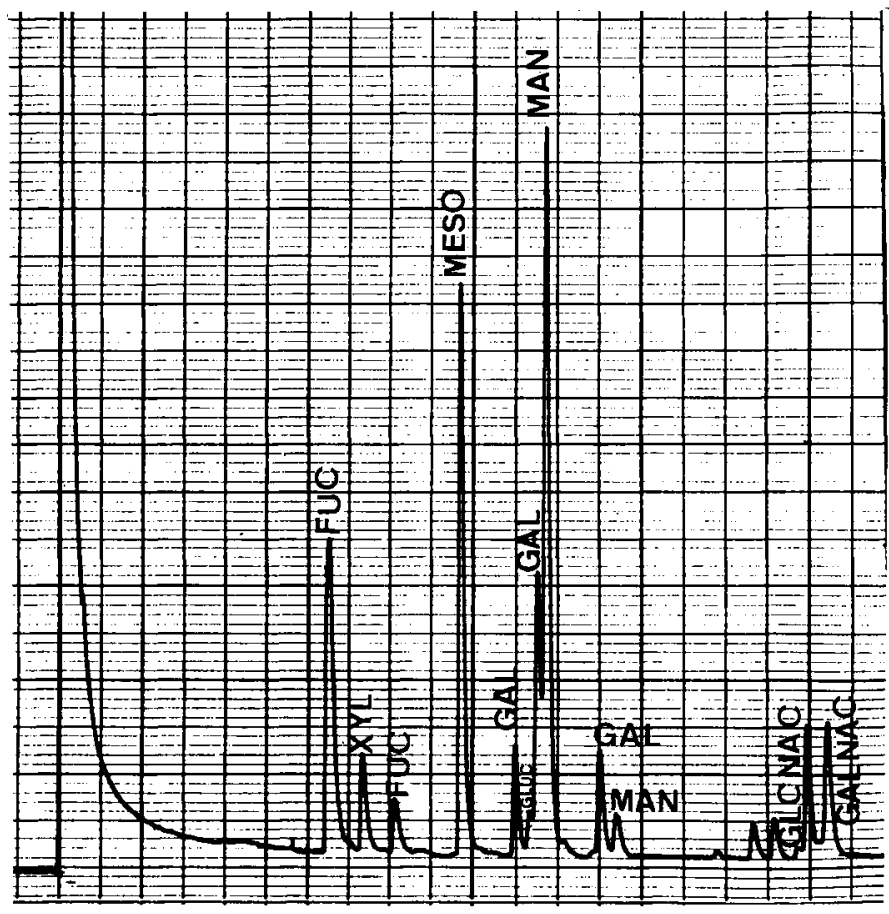

Fig 1. Analyse des glucides par chromatographie en phase gazeuse. Chromatographie des polysaccharides du clitellum mûr. II n'y a pas d'acide glucuronique ni d'acide sialique. (Fuc : fucose ; Gal : galactose ; Gluc : glucose ; Man : mannose ; Xyl : xylose; Gal/Nac : N-acéthylgalactosamine ; Glc$\mathrm{Nac}: \mathrm{N}$-acétylglucosamine). Colonne de verre $(0,3 \times 300 \mathrm{~cm})$ OV 210 ; gaz vecteur : azote à 15 $\mathrm{ml} / \mathrm{mn}$; température programmée de $100^{\circ} \mathrm{C}$ à $200^{\circ} \mathrm{C}$ à raison de $2^{\circ} \mathrm{C} / \mathrm{min}$; température de l'injecteur et du détecteur $220^{\circ} \mathrm{C}$. 
Tableau III. Teneurs en monosaccharides dans le clitellum au cours de la croissance et dans l'albumen d'un lot de 10 cocons de 1 à $10 \mathrm{j}$, valeurs exprimées en \% par rapport au poids sec (1er chiffre) et en $\%$ par rapport aux sucres totaux ( $2^{\mathrm{e}}$ chiffre en italique).

\section{Clitellums}

Immatures

Submatures

Cocons a

\begin{tabular}{|c|c|c|c|c|c|c|c|c|}
\hline \multirow[b]{2}{*}{ Fuc } & \multicolumn{2}{|c|}{ Immatures } & \multicolumn{2}{|c|}{ Submatures } & \multicolumn{2}{|c|}{ Mûrs } & \multirow[b]{2}{*}{4,0} & \multirow[b]{2}{*}{9,3} \\
\hline & 0,2 & 5,3 & 0,7 & 6,6 & 1,4 & 7,2 & & \\
\hline Gal & 0,9 & 23,7 & 2,7 & 25,7 & 3,8 & 19,5 & 8,8 & 20,4 \\
\hline Man & 0,5 & 13,2 & 1,8 & 17,1 & 6,8 & 34,9 & 12,1 & 28,1 \\
\hline Glc & 1,2 & 31,6 & 2,7 & 25,7 & 1,4 & 7,2 & 2,5 & 5,8 \\
\hline$x y l$ & 0,2 & 5,2 & 0,6 & 5,7 & 1,5 & 7,6 & 4,8 & 11,1 \\
\hline GalNac & 0,4 & 10,5 & 1,0 & 9,6 & 2,9 & 14,9 & 5,7 & 13,2 \\
\hline GlcNac & 0,4 & 10,5 & 1,0 & 9,6 & 1,7 & 8,7 & 5,2 & 12,1 \\
\hline Total & 3,8 & 100 & 10,5 & 100 & 19,5 & 100 & 43,1 & 100 \\
\hline
\end{tabular}

a Lot de cocons récoltés entre le $1^{\text {er }}$ et le $5^{\mathrm{e}} \mathrm{j}$ après la ponte.

très abondant $(28,1 \%)$ ainsi que celui $d u$ galactose $(20,4 \%)$ tandis que celui du glucose devient minoritaire $(5,8 \%)$. Les différences observées sont constamment significatives pour $P \leq 0,01$.

L'évolution des oses neutres durant l'incubation des cocons (tableau IV) montre que le glucose, presque inexistant dans les cocons frais pondus, devient relativement abondant à l'approche de l'éclosion $(12,6 \%)$. Le mannose prédomine pendant toute l'embryogenèse ( $30 \%$ environ). Le galactose est, lui aussi, bien représenté à tous les stades du développement (21 à 25\%). Dans l'ensemble, l'approche de l'éclosion s'accompagne d'une baisse générale de la teneur en glucides, à l'exception du $10^{e}$ jour où apparaît un enrichissement momentané. Juste avant l'éclosion, les glucides ne représentent plus que $14 \%$ environ du poids sec, ce qui confirme les résultats obtenus par la méthode de dosage à l'orcinol.

\section{Les lipides}

Pour des raisons matérielles, nous n'avons pas pu étudier l'évolution des teneurs en lipides au cours du développement. Aussi, nous ne donnerons ici que des résultats préliminaires partiels. Les teneurs des différentes catégories de lipides et des acides gras seront exprimées en pourcentage par rapport au poids sec. Les pourcentages relatifs seront ensuite calculés.

\section{Composition en lipides des clitellums}

Les lipides totaux, les lipides neutres et les phospholipides ont été dosés dans les clitellums aux 3 stades de maturation définis précédemment. Les résultats, reportés dans le tableau $\mathrm{V}$, montrent que les quantités de lipides sont, dans l'ensemble, relativement faibles. Néanmoins, on peut noter une légère augmentation de ces lipides, au cours 
Tableau IV. Teneurs en oses dans les cocons à différents stades d'incubation exprimées en \% par rapport au poids sec ( $1^{\mathrm{er}}$ chiffre) et en \% par rapport aux sucres totaux ( $2^{\mathrm{e}}$ chiffre en italique).

\begin{tabular}{|c|c|c|c|c|c|c|c|c|c|c|}
\hline & \multicolumn{10}{|c|}{ Albumen des cocons de } \\
\hline & \multicolumn{2}{|c|}{$1 j$} & \multicolumn{2}{|c|}{$5 j$} & \multicolumn{2}{|c|}{$10 j$} & \multicolumn{2}{|c|}{$15 j$} & \multicolumn{2}{|c|}{$20 j$} \\
\hline Fuc & 5,5 & 18,6 & 3,3 & 15,6 & 5,2 & 17,3 & 2,9 & 13,8 & 1,75 & 12,6 \\
\hline Gal & 6,6 & 22,4 & 5,2 & 24,6 & 7,0 & 23,2 & 4,4 & 20,9 & 3,2 & 23,0 \\
\hline Man & 8,9 & 30,2 & 7,0 & 33,2 & 9,3 & 30,9 & 6,1 & 28,9 & 4,2 & 30,2 \\
\hline Glc & & & 0,4 & 1,9 & & & 2,9 & 13,8 & 1,75 & 12,6 \\
\hline $\mathrm{xyl}$ & 1,3 & 4,4 & 0,8 & 3,8 & 1,2 & 4,0 & 0,7 & 3,3 & 0,5 & 3,6 \\
\hline GalNac & 4,5 & 15,3 & 2,6 & 12,4 & 4,4 & 14,6 & 2,3 & 10,9 & 1,4 & 10,1 \\
\hline GlcNac & 2,7 & 9,1 & 1,8 & 8,5 & 3,0 & 10,0 & 1,8 & 8,5 & 1,1 & 7,9 \\
\hline Total & 29,5 & 100 & 21,1 & 100 & 30,1 & 100 & 21,1 & & 13,9 & 100 \\
\hline
\end{tabular}

de la maturation, les taux passant de 8 à $10 \%$ du poids sec (différences significatives pour $p \leq 0,01$ entre ces valeurs extrêmes). Cet accroissement n'intéresse, semble-t-il, que les phospholipides, tandis que les quantités de lipides neutres restent à peu près stationnaires, entre les 2 tiers et 4 cinquièmes des lipides totaux. Malgré un tri- plement de leur teneur, les phospholipides demeurent donc en très faible quantité. Quant aux glycolipides et résidus divers, ils présentent une diminution importante au moment de la maturation par rapport au stade immature. Les valeurs demeurent toutefois faibles, de même que celles des phospholipides.

Tableau V. Teneurs en lipides du clitellum d'Eisenia fetida à différents stades de maturation.

\section{Clitellums}

Immatures

Submatures

Mûrs

$\%$ du poids sec

Lipides totaux

Lipides neutres

Phospholipides

Glycolipides + résidus a

$$
\begin{gathered}
8,0 \pm 1,5 \\
6,0 \pm 0,7 \\
0,8 \pm 1,7 \\
1,2
\end{gathered}
$$
$9,0 \pm 1,8$
$7,3 \pm 1,4$
$1,1 \pm 1,5$
0,6
$10,0 \pm 1,8$
$6,6 \pm 1,6$
$2,7 \pm 1,3$
0,7

$\%$ des lipides totaux

Lipides neutres

75,0

81,1

66,0

Phospholipides

10,0

12,2

27,0

Glycolipides + résidus a

15,0

6,6

7,0

a Valeurs calculées par différence. 
Tableau VI. Teneurs en acides gras dans les clitellums à différents stades de maturation et dans l'albumen des cocons.

\begin{tabular}{lcccc} 
Acides gras totaux & \multicolumn{3}{c}{ Clitellums } & Cocons \\
& Immatures & Submatures & Mûrs & \\
& & & & \\
& & $5,4 \pm 1,2$ & $3,6 \pm 0,9$ & $10,6 \pm 1,7$ \\
\% du poids sec & $3,5 \pm 1,1$ & $73,9 \pm 16,4$ & $54,5 \pm 13,6$ & - b
\end{tabular}

a Lot de cocons récoltés entre le $1^{e r}$ et le $5^{e} \mathrm{j}$ après la ponte; ${ }^{b}$ les lipides neutres n'ont pas été dosés dans les cocons.

\section{Analyse des acides gras dans les clitellums et dans les cocons}

Après saponification des lipides neutres, les acides gras ont été dosés dans les clitellums immatures, submatures et mûrs ainsi que dans l'albumen d'un lot de cocons âgés de 5 à $10 \mathrm{j}$. Sur le tableau VI sont reportées les teneurs totales en acides gras exprimées en pourcentage de la matière sèche et en pourcentage des lipides neutres. La légère augmentation des quantités totales d'acides gras au cours de la phase submature par rapport aux stades immatures ou mûrs n'est pas statistiquement significative, à cause de la valeur élevée des écarts types. En revanche, la différence est significative entre les taux d'acides gras des cocons et ceux des clitellums $(10,6$ vs 3,5 à $5,4 \%)$. Ceci pourrait traduire une mise en réserve relativement importante de substances lipidiques dans l'albumen des cocons.

La composition en acides gras des lipides neutres des clitellums et de l'albumen de cocons est reportée dans le tableau VII, d'une part, en précisant les stades d'évolution des clitellums, et d'autre part, en séparant les acides gras saturés des insaturés. Les résultats sont exprimés en pourcentage de matière sèche et en pourcentage des acides gras totaux (chiffres en italique). Les acides gras à chaîne très courte (moins de $14 \mathrm{C}$ ), volatils, n'ont pu être décelés par la méthode utilisée. Un exemple de chromatogramme relatif aux clitellums mûrs est donné sur la figure 2.

À tous les stades de maturation des clitellums et dans l'albumen des cocons, on note l'existence simultanée d'acides gras saturés et d'acides gras insaturés. Le rapport i/s (insaturés par rapport aux saturés) présente des valeurs dont la variation est intéressante : 0,64 chez les immatures vs environ 1 pour les autres stades et chez les cocons, ce qui semble indiquer une modification importante de la constitution des lipides au moment de la maturation du clitellum, à la puberté.

Sur 16 acides gras détectés, une douzaine sont en quantité notable, les 4 plus importants étant l'acide palmitique (16:0) et l'acide stéarique (18:0) parmi les saturés, l'acide oléique (18:1) et l'acide vaccénique (18:1) parmi les insaturés. On retrouve en général les mêmes acides gras à tous les stades du clitellum et dans l'albumen des cocons, mais dans des proportions variant d'une manière parfois importante. Le rapport 18:1 oléique / 18:1 vaccénique présente une variation importante au moment de la maturation : sa valeur $(2,2)$ est divisée par 2. Ceci confirme la modification de 
la composition des lipides au moment où se développe le clitellum. En ce qui concerne l'albumen des cocons, on peut remarquer, en plus d'une quantité d'acides gras très nettement supérieure $(10,6 \%$ du poids sec), une répartition des acides gras assez différente : l'acide palmitoléique (16:1) $y$ est plus abondant, tandis que les acides palmitique (16:0) et stéarique (18:0) y sont nettement moins bien représentés. Enfin, on peut noter, dans tous les échantillons, la présence de l'acide margarique (17:0) en quantité non négligeable (de 5 à 10\%). Ainsi, Eisenia est l'un des rares animaux à posséder des acides gras à nombre impair de carbones.

Tableau VII. Composition en acides gras des lipides neutres des clitellums et de l'albumen des cocons.

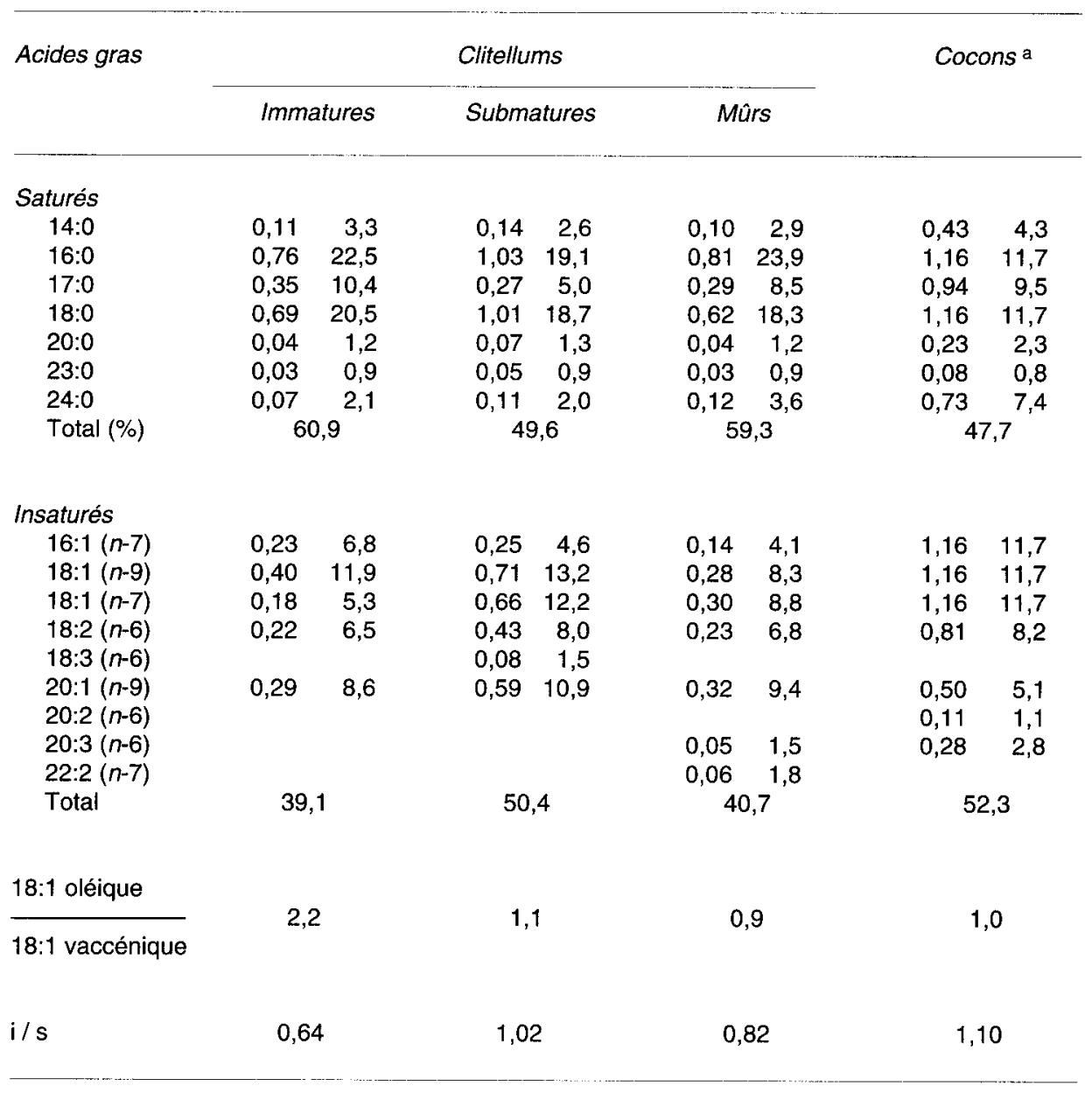

a Lot de cocons récoltés entre le $1^{\text {er }}$ et le $5^{e} \mathrm{j}$ après la ponte. 
Fig 2. Analyse des lipides par chromatographie en phase gazeuse. Chromatogramme des acides gras du clitellum mûr. Colonne capillaire de silicone OV 101 (85 m x 0,35 mm ID) ; pression du gaz vecteur (hélium) : 0,4 bar ; température programmée de 100 à $200^{\circ} \mathrm{C}$ à raison de $2^{\circ} \mathrm{C}$ par min ; température de l'injecteur et du détecteur : $240^{\circ} \mathrm{C}$.

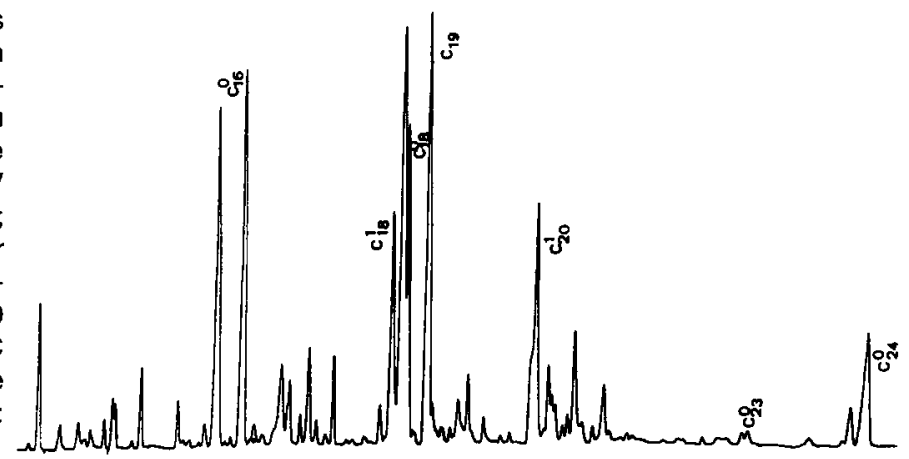

\section{DISCUSSION ET CONCLUSION}

L'analyse qualitative des glucides montre que l'on retrouve toujours les mêmes monosaccharides quels que soient les stades de la clitellogenèse ou du développement des embryons. Ces mêmes glucides, ainsi que la galactosamine et la glucosamine, ont d'ailleurs été détectés dans les ovocytes de l'annélide polychète Perinereis cultrifera (Porchet, 1974). Le rhamnose, présent dans les ovocytes de cette espèce, est, en revanche, absent de l'albumen du cocon d'Eisenia. L'acide sialique n'a été détecté ni chez $E$ fetida ni dans les ovocytes de $P$ cultrifera. Sur le plan quantitatif, l'ovocyte de Nereis est beaucoup moins riche en glucides $(\leq 23 \%)$ que le cocon d'Eisenia qui en compte environ 2 fois plus. L'évolution de la teneur glucidique dans le cocon à l'approche de l'éclosion est en relation avec le développement embryonnaire. L'augmentation relative observée au $10^{e}$ jour correspond au moment du maximum d'absorption de l'albumen dans l'archentéron, au cours duquel des actions enzymatiques permettent sa liquéfaction. Mais les oses étant les réserves énergétiques de l'embryon, il est normal d'en observer une diminution de la teneur pendant toute l'ontogenèse. Tous les glucides sont concernés par cette baisse, à l'exception du glucose dont le taux augmente légèrement.
Depuis les travaux de Grove et Cowley (1928), on admet que l'albumen est sécrété par les "cellules à fines granulations" du clitellum. Nos analyses ont montré la forte teneur en glucides de l'albumen (40 à $50 \%$ du poids sec). On doit donc pouvoir les déceler au niveau du site de synthèse. Or, malgré une étude histochimique poussée, Defretin et Demailly (1953) n'ont pas pu mettre en évidence de polysaccharides dans ce type de cellules. Toutefois Herlant-Meewis (1958-1959) observe que les "cellules de la couche profonde" se colorent fortement à l'APS chez Eisenia. Morris (1983) décèle dans les cellules à fins granules d' $E$ fetida des composés glycoprotéiques. Richards (1977) obtient chez le dulcicole Lumbricillus rivalis une réaction faiblement positive à l'APS, au niveau des "cellules globulaires", lieu d'élaboration du contenu du cocon chez cette espèce. Malécha et Vinckier (1983) détectent une réaction fortement positive à I'APS dans la sécrétion constituant les réserves du cocon (cellules de type 4) de la sangsue Piscicola. Nos dosages biochimiques ont été effectués sur la totalité du clitellum, sans pouvoir séparer les différents types cellulaires. Ils montrent un accroissement global des glucides dans cet organe au cours de la puberté, mais ils ne permettent pas de préciser les sites de synthèse. II est vraisemblable, d'ailleurs, que toutes les cellules de cet épithélium effectuent des synthèses de glu- 
cides libres ou liés dans des glycoconjugués.

Bien qu'impliqué dans la synthèse des substances de réserve, le clitellum d'Eisenia contient assez peu de lipides (8 à 10\% du poids $\mathrm{sec}$ ). Les valeurs obtenues sont toutefois comparables à celles trouvées par Hansen et Czochanska (1975) (de 8,5 à $11 \%$ suivant les saisons) chez Lumbricus rubellus et Allolobophora caliginosa des prairies de Nouvelle-Zélande. En revanche, ces valeurs sont nettement supérieures à celles citées par Whitten et Goodnight (1966) pour Lumbricus terrestris et Eisenia rosea (respectivement 6,05 et 4,5 ). Un chiffre encore plus bas est obtenu par McLaughlin (1971) chez $E$ fetida mûr : $2,25 \%$ du poids sec. À l'inverse, Durchon et Lafon (1951) avaient obtenu $17,3 \%$ de lipides chez $L$ terrestris. II parait donc y avoir d'assez notables écarts, dans une même espèce ou dans les espèces voisines, suivant les auteurs. Mais ces recherches de lipides ont porté sur la totalité du corps des animaux, incluant aussi bien les tissus et organes que le matériel ingéré et les microorganismes du tube digestif. En revanche, nos analyses se sont portées spécifiquement sur le clitellum, isolé du reste du corps, à l'exception d'une relativement mince couche musculaire. Par ailleurs, les techniques de dosage, quelque peu différentes, sont peut-être en cause, mais aussi, sans doute, l'état métabolique ou l'état génital des vers, non précisé le plus souvent par les différents auteurs. Chez les polychètes errantes, Durchon et Lafon (1951) observent des quantités de lipides variant, suivant les espèces, de 9 à $15,5 \%$ et, chez les sédentaires, de 6,7 à 14,6\%. De son côté, Karnovsky (1969) rapporte des valeurs du même ordre pour quelques espèces. Plus récemment, Fontaine (1982) dose chez $P$ cultrifera les lipides totaux du liquide cœlomique, des cœlomocytes et des ovocytes en fonction de l'âge des vers. II trouve que les lipides constituent 12,5 à $26,5 \%$ du poids sec. Les polychètes semblent donc légèrement plus riches en lipides que les lombriciens. Les oligochètes limicoles Tubifex tubifex et Limnodrilus hoffmeisteri (Whitten et Goodnight, 1966) auraient des teneurs en lipides de l'ordre de 17 à $25 \%$, donc nettement supérieures à celle des terricoles. Les lipides neutres représentent, chez $E$ fetida, de 66 à $81 \%$ des lipides totaux, tandis que les phospholipides ne correspondent qu'à 10 à $27 \%$. Ces résultats s'accordent avec ceux de McLaughlin (1971) qui obtient 11\% de phospholipides sur la même espèce, ou ceux de Cerbulis et Taylor (1969) qui indiquent $25 \%$ de phospholipides chez le lombric. En revanche, ils ne concordent pas avec ceux de Hansen et Czochanska (1975) qui mesurent 50 à $63 \%$ de phospholipides chez les lombriciens $L$ rubellus et $A$ caliginosa, taux qui semble considérable. Dans nos résultats, malgré la faiblesse des teneurs observées, il faut souligner l'augmentation importante des phospholipides lors de la maturation du clitellum. Une part de ces phospholipides entre, peut-être, dans la constitution de lipoprotéines de réserve, comme chez les insectes (Chino et al, 1969) ou chez les crustacés (Lee et Puppione, 1978).

Les acides gras présentent une large répartition, comme chez toutes les annélides étudiées. Sur le plan qualitatif, les acides gras présents dans les clitellums et dans les cocons appartiennent à différentes voies métaboliques. Ce sont les mêmes acides qui ont été mis en évidence dans l'ovocyte de $P$ cultrifera (Fontaine, 1982). Bien que les lipides des animaux ne contiennent pratiquement que des acides gras à nombre pair d'atomes de carbone, ceux d'Eisenia possèdent de l'acide margarique (17:0) en quantité non négligeable (entre 5 et $10 \%$ ) et des traces d'acide tricosanoïque (23:0). Ces acides gras se retrouvent aussi dans les analyses de Hansen et Czochanska (1975). De leur côté, Stefanovic et Djurdjic (1977), remarquant une abondance 
Tableau VIII. Teneurs, en \% du poids sec, en glucides, lipides et protéines, des clitellums immatures, submatures et mûrs.

\section{Clitellums}

Immatures Submatures Mûrs

\begin{tabular}{|c|c|c|c|}
\hline Glucides totaux & 4,5 & 11,8 & 21,0 \\
\hline Lipides totaux & 8 & 9 & 10 \\
\hline Protéines totales a & 79 & 71 & 59 \\
\hline Résidus ${ }^{\circ}$ & 8,5 & 8,2 & 10 \\
\hline
\end{tabular}

a Chiffres provenant de Rouabah-Sadaoui et Marcel (1995) : méthodes de Geiger et Bessman ; b calculés par différence.

d'acides gras à nombre impair de carbones, estiment que les vers de terre dévient des autres animaux par rapport au spectre habituel des acides gras. L'acide margarique, ainsi que les autres acides à nombre impair de carbones, sont absents chez $P$ cultrifera (Fontaine, 1982). Les acides gras les plus abondants sont, dans l'ordre, l'acide palmitique et l'acide stéarique, pour les saturés, l'acide oléique et l'acide vaccénique, pour les insaturés. On retrouve ces acides, avec des teneurs légèrement différentes, dans les études de Hansen et Czochanska (1975). Les variations du rapport des acides gras insaturés aux acides gras saturés sont intéressantes (tableau VII). Ce rapport augmente d'une manière caractéristique lors de la maturation, passant de 0,64 à 1,10 , augmentation corrélative d'un allongement des chaînes carbonées. Le calcul de ce même rapport $\mathrm{i} / \mathrm{s}$ effectué à partir des chiffres fournis par Hansen et Czochanska montre, en revanche, des variations saisonnières peu interprétables : de 0,67 à 1,59 au printemps à 2 ans d'intervalle, maximum en hiver $(2,0)$, valeur intermédiaire à l'automne. Toutefois, les valeurs obtenues sont du même ordre de grandeur que les nôtres.

Les glucides sont donc le composant majoritaire de l'albumen des cocons (tableau
VIII), ce qui explique la richesse énergétique des réserves de l'embryon. Ces glucides sont, au moins en partie, associés aux protéines $B$ et $C$ et à la lipoprotéine $A$ (Rouabah-Sadaoui et Marcel, 1995). II sera nécessaire maintenant d'étudier en détail les variations des taux des glucides et lipides pendant le développement embryonnaire pour les comparer à celles des protéines, avant d'envisager le contrôle neurohormonal de la production des glycoprotéines et en particulier de la synthèse de la «vitellogénine» mise en évidence précédemment (Rouabah-Sadaoui et Marcel, 1995).

\section{RÉFÉRENCES}

Cerbulis J, Taylor MW (1969) Neutral lipids and fatty acid composition of earthworms (Lumbricus terrestris). Lipids 4, 363-368

Chino H, Murakami S, Harashima K (1969) Diglyceridecarrying lipoproteins in insect hemolymph. Isolation, purification and properties. Biochem Biophys Acta $176,1-26$

Dawydoff $C$ (1959) Ontogenèse des annélides. In : Traité de zoologie, V (PP Grasse, ed), Masson, 594-686

De Jorge FB, Sawaya MC (1967) Comparative biochemical studies on the Oligochaetes Pheretima hawayana, Glossoscolex grandis and Rhinodrilus sp. Comp Biochem Physiol 22, 359-369

De Jorge FB, Haeser PE, Ditadi ASF, Petersen JA Ulhoa Cintra AB, Sawaya P (1965) Histochemical 
studies on the giant earthworm Glossoscolex giganteus (Leuckart). Comp Biochem Physiol 16, 491 496

Defretin R, Demailly E (1953) Sur quelques caractères histochimiques des divers types de cellules glandulaires du clitellum du lombric. CR Soc Biol 147, 12511253

Devriès $J$ (1973) La formation et la destinée des feuiliets embryonnaires chez le lombricien Eisenia foetida (annélide oligochète). Arch Anat Micr Morph Exp 62, 15-38

Durchon M, Lafon M (1951) Quelques données biochimiques sur les annélides. Ann Sc Nat Zool, $11^{\mathrm{e}} \mathrm{S}, 13$ 427-451

Folch J, Less M, Stanley GH (1957) A simple method for the isolation and puritication of total lipids from animal tissues. J Biol Chem 226, 497-509

Fontaine $F(1982)$ Les lipides cœlomiques chez $P e$ rinereis cultrifera Grübe. Thèse doctorat $3^{e}$ cycle, Lille-I, $130 \mathrm{p}$

Grove AJ, Cowley LF (1928) The relation of the glandular elements of the clitellum of the brandling worm (Eisenia foetida Sav) to the secretion of the cocoon. Quart J Microsc Sci 71, 31-45

Hansen P, Czochanska Z (1975) The fatty acid composition of the lipids of earthworm. J Sci Fd Agric 26, $961-971$

Herlant-Meewis H (1958-1959) Évolution des caractères sexuels au cours de la croissance et de la reproduction chez Eisenia foetida. Ann Soc Roy Zool Belg $89,281-336$

Karnovsky ML (1969) Annelida, Echiurida and Sipunculida - lipid components and metabolism. In : Chemical Zoology (M Florkin, BT Scheer, eds), Vol IV, Acad Press, New York, NY, USA 205-210

Lechenault $H$ (1968) Étude cytochimique et ultrastructurale de l'ovocyte d'Eisenia foetida Sav. ZZelforsch 90, 96-112

Lee RF, Puppione DL (1978) Serum lipoproteins in the Spiny lobster, Panulinus interruptus. Comp Biochem Physiol 59B, 239-243
Malécha J, Vinckier D (1983) Formation du cocon chez I'hirudinée rhynchobdelle Piscicola geometra L. Arch Biol 94, 183-205

McLaughlin J (1971) Biochemical studies on Eisenia foetida (Savigny, 1826), the brandling worm. I. Tissue lipids and sterols. Comp Biochem Physiol 38 , 147-163

Montreuil J, Spik G (1963) Microdosage des glucides. Fasc 1. Méthodes colorimétriques de dosages des glucides totaux. Lab chimie biol, fac sci éd), Lille, $116 \mathrm{p}$

Morris GM (1983) The cocoon producing cells of Eisenia foetida (Annelida, Oligochaeta); a histochemical and ultrastructural study. J Morph 177, 41-50

Porchet M (1974) Activité endocrine cérébrale chez les Nereidae. Thèse doct ès-sci, Lille

Richards KS (1977) The immunochemistry and ultrastructure of the clitellum of the enchytraeid Lumbricillus rivalis (Oligochaeta: Annelida). J Zool 183, 161-176

Rouabah-Sadaoui L (1984) Contribution à l'étude biologique et biochimique du clitellum et du cocon d' $\mathrm{E}$ senia fetida Sav (annélide oligichète). Thèse $3^{e}$ cycle, Lille

Rouabah-Sadaoui L, Marcel R (1995) Analyse des réserves nutritives protéiques dans le clitellum et dans l'albumen du cocon chez Eisenia fetida Sav (Annélide, Oligochète). Mise en évidence d'une glycolipoprotéine comparable à une vitellogénine. Reprod Nutr Dévelop 35, 491-501

Stefanovic GJ, Djurdjic V (1977) An investigation on lipids isolated from Lumbricus terrestris. Bull Acad Serve Sci Arts, Cl Sci Nat \& Math, Sci Nat ${ }^{\circ} 15$; 85-89

Whitten BK, Goodnight CJ (1966) The comparative chemical composition of 2 aquatic oligochaetes. Comp Biochem Physiol 17, 1205-1207

Zanetta JP, Breckendrige WC, Vincendon G (1972) Analysis of monosaccharides by gas-liquid chromatography of the $O$-methylglycosides as trifluoroacetate derivatives. Application to glycoproteins and glycolipids. J Chromatogr 69, 291-304 